\title{
Aboard the ISS: intersubunit signaling revealed in the p97 ATPase
}

\author{
A new cryo-EM study reveals how ATP hydrolysis in the p97 ATPase is coordinated to unfold the protein's \\ substrates and provides new foundations for the design of small molecule therapeutics targeting p97 in cancer, \\ neurodegeneration and viral infection.
}

\section{Edward C. Twomey}

$\mathrm{P}$ rotein homeostasis is critical for proteome maintenance and thus for life. In principle, protein turnover is carried out by the proteasome, which degrades proteins marked by polyubiquitin into their constituent amino acids, allowing them to be recycled. However, the proteasome needs an unstructured polypeptide stretch to process its polyubiquitinated substrates ${ }^{1}$. Therefore, proteins that are highly-ordered, within tight complexes or embedded in membranes are not directly accessible for degradation, even if they are tagged by polyubiquitin. For these substrates, the p97 ATPase must first unfold and segregate the polyubiquitinated protein so that it can be recognized and degraded by the proteasome. A prime example of this process is endoplasmic reticulum (ER)-associated protein degradation, or ERAD ${ }^{2}$; here, $\mathrm{p} 97$ recognizes polyubiquitinated proteins displayed on the ER membrane, unfolds them and extracts them into the cytosol for proteasomal degradation ${ }^{3}$. The same principles hold for protein homeostasis across different organelles and various other cellular processes, for example, $\mathrm{p} 97$ is required for chromatin-associated protein degradation, mitochondrial-associated degradation, ribosome-associated degradation, autophagy, and so on. These topics have been wonderfully reviewed recently by Johannes van den Boom and Henmo Meyer ${ }^{4}$. What unites most of these processes is the protein substrate's polyubiquitin signal, which is first recognized and unfolded by the ATPase 5 . The importance of $\mathrm{p} 97$ cannot be understated, as it is essential for many forms of life, from single-celled yeast to humans. Therefore, targeting p97 with small molecules is a rapidly expanding therapeutic approach to target cancers, neurodegenerative diseases and viral infections (reviewed by Donna Huryn and colleagues $\left.{ }^{6}\right)$. Especially timely, a small molecule inhibitor of p97 has recently been identified as a potential drug against SARS-CoV-2 (ref. ${ }^{7}$ ). a

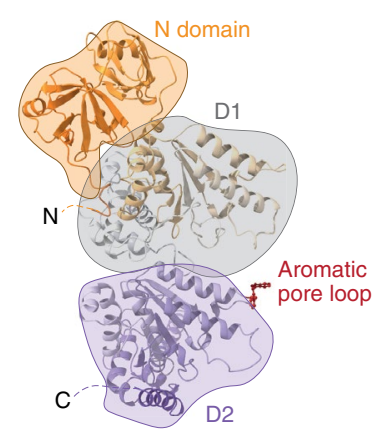

c

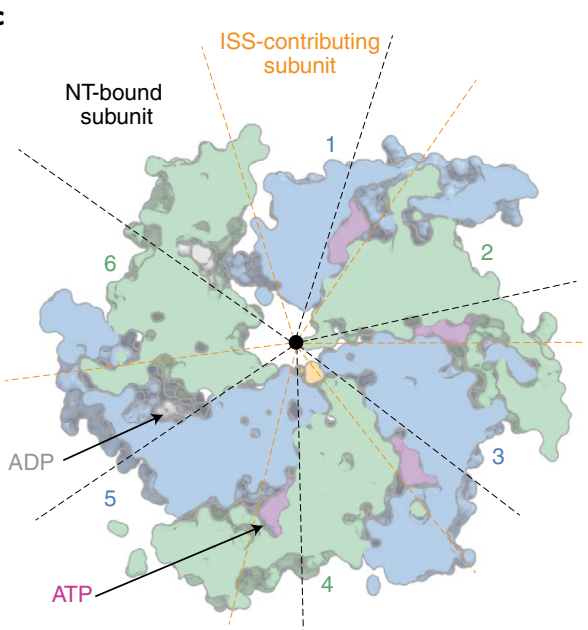

d

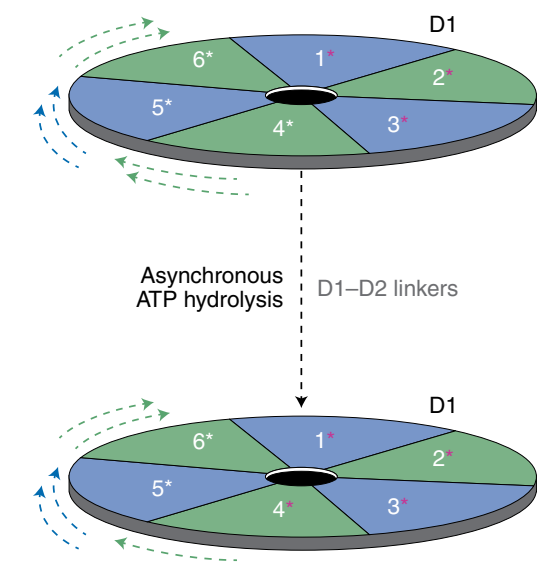

Fig. 1 | Intersubunit signaling in the $\mathbf{p} 9 \mathbf{7}$ ATPase. a, Arrangement of a $\mathrm{p} 97$ protomer from $\mathrm{N}$ terminus ( $\mathrm{N}$, orange) to $\mathrm{C}$ terminus (C, purple). b, The p97 hexamer unfolding the substrate (yellow), both shown as surfaces. Alternating subunits are colored in blue and green, respectively. Two subunits are removed to show the central pore. c, Slice through the center of D2 in the p97 hexamer, colored as in $\mathbf{b}$, with ATP in magenta and ADP in gray. Subunits are numbered 1 through 6. Dashed lines show the NT-bound subunit (black) and ISS-contributing subunit (orange). d, Proposed mode of asynchronous ATP hydrolysis to drive communication between D1-D2 and subunits, which are ADP-bound (white asterisk) or ATP-bound (magenta asterisk).

The p97 ATPase belongs to the AAA+ family (ATPases associated with diverse cellular activities). These proteins form multimeric assemblies and use ATP hydrolysis to exert force on substrate proteins $^{8}$. $\mathrm{p} 97$ is a member of the type II AAA+ ATPase family, and p97 monomers consists of $\mathrm{N}$ domains at the $\mathrm{N}$ terminus, 
followed by two ATPase-containing domains, D1 and D2 (Fig. 1a). A distinct pore loop with two aromatic residues, W551 and F552, chelates unfolded polypeptides to pull them through the pore of the p97 hexamer, its functional unit. The original discovery of p97 and the observation of this structural arrangement was aided by the abundance of p97 in Xenopus laevis oocytes, as negative-stain electron microscopy of p97 purified from this source revealed an oligomer with hexameric radial symmetry ${ }^{9}$. Since these original images, the structurefunction relationship in p97 was analyzed using both $\mathrm{X}$-ray crystallography and cryo-electron microscopy (cryo-EM) ${ }^{10-14}$. Although these works were seminal in the initial understanding of $\mathrm{p} 97$, in these structures, p97 was captured in the absence of substrate and showed perfectly symmetric assemblies, reminiscent of the first p97 images. Yet as we know, biology occurs when symmetry breaks.

In this issue of Nature Structural \& Molecular Biology, Pan et al. ${ }^{14}$ show that the p97 hexamer breaks symmetry and pinches around the unfolded substrate as it is unfolded (Fig. 1b). In this state, the aromatic pore loop in D2 is arranged like a spiral staircase. While this staircase mechanism is now a common theme for protein unfolding across AAA+ ATPases, the work from Pan et al. reveals how intersubunit communication is coordinated and, importantly, how this coordination may influence intrasubunit communication between the $\mathrm{D} 1$ and $\mathrm{D} 2$ rings to drive substrate translocation through the ATPase pore. In AAA+ ATPases, an intersubunit signaling (ISS) motif is critical for coordinating the nucleotide (NT) states of adjacent subunits ${ }^{12,15,16}$. The authors observe a new conformation in which the ISS extends into the NT-binding site of a neighboring subunit, thereby precluding ATP hydrolysis and ADP release. Therefore, the ISS must be retracted for the pore loop staircase to move. This observation provides new context for the original work on AAA+ ISS sites, wherein the ISS was proposed to be a communication module that detects the NT state of the neighboring subunit. This can best be visualized by looking down through the pore axis at the p97 D2, where the space between the NT-bound subunit and the ISS-contributing subunit increases as the NT state changes to decouple the ISS from the ATP-binding pocket (Fig. 1c). What implications does this have for $\mathrm{p} 97$ signaling?
For one, the neighboring ISS must be removed from the ATP-binding pocket for hydrolysis to occur. Based on these structures $^{14}$, cryo-EM studies on Cdc48, the yeast homologue of p97, and the archaeal homologue VAT, ATP hydrolysis is expected to occur sequentially in subunits $s^{5,17,18}$. Thus, in the context of the new findings provided by Pan et al., the ISS of subunit 5, for example, must retract from subunit 4 so that hydrolysis and NT exchange can occur in subunit 4 (Fig. 1c). The unprecedented asynchronous ATP-hydrolysis states of D1 and D2 in the same subunit observed by Pan et al. provide insights into how this may occur ${ }^{14}$. For example, the authors observe ADP in the D1 domain of subunit 4 , but ATP in D2. This suggests that these data reveal an active processing state. The structure of the Cdc48 initiation complex, on the other hand, has a planar, ATP-bound, but hydrolysis-competent, D1 and a staircase in D2. Asynchronous ATP hydrolysis states have also not been observed in the proposed processing states of Cdc48 with ADP- $\mathrm{BeF}_{\mathrm{X}}{ }^{5,17}$, which perturbs NT hydrolysis and locks the angles between D1 and D2 and thus blocks substrate translocation. I would like to propose that asynchronous hydrolysis between D1 and D2 could prime the D2 subunit for ATP hydrolysis and NT exchange. This model is illustrated in Fig. 1d, wherein the D1 domain of subunit 4 is ADP-bound. This is communicated to D2 through the D1-D2 linkers within the subunit, which is primed for ATP hydrolysis through the removal of the ISS motif of subunit 5 . This results in the removal of the ISS motif of subunit 4 from the ATP binding sites of subunit 3 (Fig. 1c, subunits 5 and 6 in D2). This provides a working model for intrasubunit and intersubunit communication and for moving the ATPase staircase. The caveat is that while the data reported by Pan et al. show that ATP hydrolysis in D1 is necessary for substrate translocation, D1 does not appear to be as critical in Cdc48 (ref. ${ }^{19}$ ). The transition from initiation ${ }^{5}$ to translocation ${ }^{14}$ would probably require dislodging of the p97 cofactor ${ }^{20}$.

The importance of the ISS in NT hydrolysis is also exemplified by cryo-EM data on p97 in complex with the p97-inhibitor ${ }^{6}$ NMS-873 (ref. ${ }^{14}$ ), which is a candidate drug for cancer treatment and antiviral therapies. NMS-873 locks in the ISS motif, allosterically inhibiting substrate translocation. Coupled with cryo-EM data reported by Subramaniam and colleagues ${ }^{13}$ on p97 in complex with another small molecule inhibitor, UPCDC30245, these studies spearhead exciting times for p97 drug design.

Despite the wealth of new information on p97, many intriguing questions remain. Perhaps one of the most interesting ones is how p97 interfaces with the proteasome. Does p97 directly interact with the catalytic subunits of the proteasome to form a proteolytic machine $e^{21}$, or is there rapid hand off after ubiquitin unfolding, as evidenced by association of the proteasome with unstructured polypeptides on the ER surface $^{22}$ ? Stay tuned as the stories unfold.

\section{Edward C. Twomey (D) 1,2凶}

${ }^{1}$ Department of Biophysics and Biophysical Chemistry, Johns Hopkins University School of Medicine, Baltimore, MD, USA. ${ }^{2}$ The Solomon $H$. Snyder Department of Neuroscience, Johns Hopkins University School of Medicine, Baltimore, MD, USA.

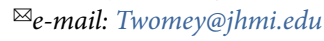

Published online: 14 July 2021 https://doi.org/10.1038/s41594-021-00622-5

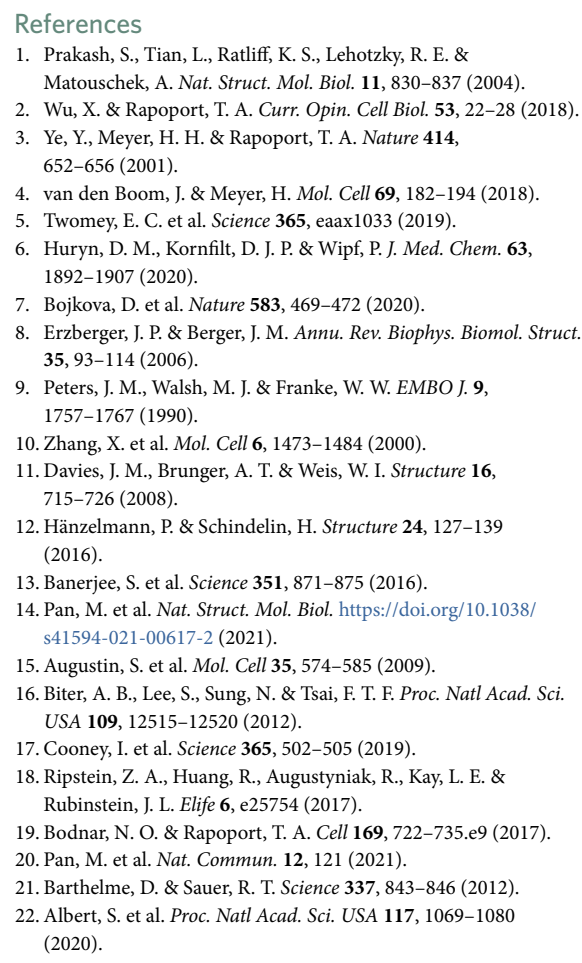

12. Hänzelmann, P. \& Schindelin, H. Structure 24, 127-139 (2016).

13. Banerjee, S. et al. Science 351, 871-875 (2016).

14. Pan, M. et al. Nat. Struct. Mol. Biol. https://doi.org/10.1038/ s41594-021-00617-2 (2021).

15. Augustin, S. et al. Mol. Cell 35, 574-585 (2009).

16. Biter, A. B., Lee, S., Sung, N. \& Tsai, F. T. F. Proc. Natl Acad. Sci. USA 109, 12515-12520 (2012).

17. Cooney, I. et al. Science 365, 502-505 (2019).

18. Ripstein, Z. A., Huang, R., Augustyniak, R., Kay, L. E. \& Rubinstein, J. L. Elife 6, e25754 (2017).

19. Bodnar, N. O. \& Rapoport, T. A. Cell 169, 722-735.e9 (2017).

20. Pan, M. et al. Nat. Commun. 12, 121 (2021).

21. Barthelme, D. \& Sauer, R. T. Science 337, 843-846 (2012)

22. Albert, S. et al. Proc. Natl Acad. Sci. USA 117, 1069-1080 (2020).

Acknowledgements

The author thanks Johns Hopkins University School of Medicine for support. Figures were made with UCSF ChimeraX and Adobe Illustrator.

\section{Competing interests}

The author declares no competing interests. 